Ostermann, F.; Woszidlo, R.; Nayeri, C. N.; Paschereit, C. O.

\title{
Phase-Averaging Methods for the Natural Flow Field of a Fluidic Oscillator
}

Journal article | Accepted manuscript (Postprint)

This version is available at https://doi.org/10.14279/depositonce-8172

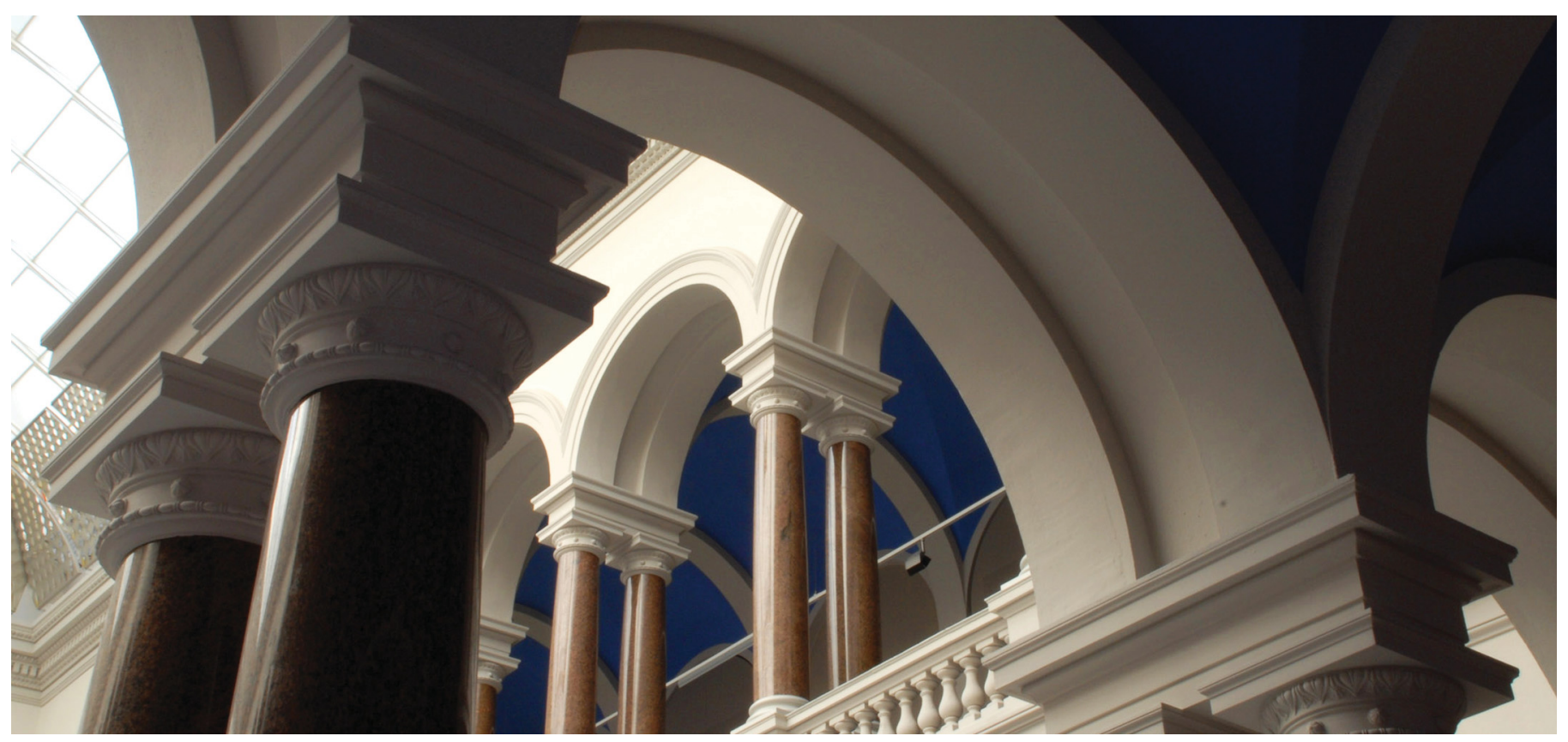

Ostermann, F., Woszidlo, R., Nayeri, C. N., \& Paschereit, C. O. (2015). Phase-Averaging Methods for the Natural Flowfield of a Fluidic Oscillator. AIAA Journal, 53(8), 2359-2368. https://doi.org/10.2514/1.j053717 


\title{
Phase-Averaging Methods for the Natural Flow Field of a Fluidic Oscillator
}

\author{
Florian Ostermann* \\ Technische Universität Berlin, Berlin, 10623, Germany \\ Rene Woszidlo ${ }^{\dagger}$ \\ The University of Kansas, Lawrence, KS 66045, USA \\ Christian N. Nayeri ${ }^{\ddagger}$ and Christian O. Paschereit ${ }^{\S}$ \\ Technische Universität Berlin, Berlin, 10623, Germany
}

\begin{abstract}
The presented study examines various methods for phase-averaging the naturally oscillating flow field of a scaled-up fluidic oscillator. No external trigger is employed to control the oscillation of the flow. Mathematical and signal conditioning approaches for phase-averaging the data are categorized and described. The results of these methods are evaluated for their accuracy in capturing the natural flow field. The respective criteria are based on the minimum fluctuation in oscillation period length, the conservation of velocity amplitudes, and the number of snapshots per phase-averaging window. Although all methods produce reasonable qualitative results, only two methods are identified to provide the desired quantitative accuracy and suitability for the investigated flow field. The first method is based on conditioning a time-resolved pressure signal from the feedback channels in the oscillator. An autocorrelation applied to the reference signal improves the period identification. The second method employs a mathematical approach by means of proper orthogonal decomposition. Because the conventional use of POD reveals shortcomings in quantitative accuracy, it is modified by imposing an even distribution of snapshots per phase angle window. The results demonstrate the feasibility and improved accuracy of the modified POD. Therefore, accurate phase-averaging can be conducted without the need for a time-resolved reference signal.
\end{abstract}

\section{Nomenclature}

modal coefficient

hydraulic diameter, $\mathrm{m}$

Fourier transform

reference signal

Hilbert transform

pressure, $\mathrm{Pa}$

time, $s$

velocity magnitude, $\mathrm{m} / \mathrm{s}$

velocity in $x$-direction, $\mathrm{m} / \mathrm{s}$

velocity in $y$-direction, $\mathrm{m} / \mathrm{s}$

Cartesian $\mathrm{x}$ coordinate, $\mathrm{m}$

$\begin{array}{ll}y & \text { Cartesian y coordinate, } \mathrm{m} \\ \mathrm{R} & \text { correlation matrix } \\ \mathrm{sgn} & \text { signum function } \\ a & \text { analytical } \\ \text { outlet } & \text { property at the oscillator outlet } \\ \lambda & \text { eigenvalue } \\ \omega & \text { angular frequency } \\ \Phi & \text { decomposed mode } \\ \phi & \text { phase angle, }{ }^{\circ}\end{array}$

y Cartesian y coordinate, $\mathrm{m}$

a analytical

outlet property at the oscillator outlet

eigenvalue

angular frequency

phase angle, ${ }^{\circ}$

\footnotetext{
*PhD Student, Hermann-Föttinger-Institut, AIAA Student Member

$\dagger$ Visiting Assistant Professor, Aerospace Engineering, AIAA Member.

${ }^{\dagger}$ Research Associate, Hermann-Föttinger-Institut, AIAA Member.

$\S$ Professor, Hermann-Föttinger-Institut, AIAA Senior Member.
} 


\section{Introduction}

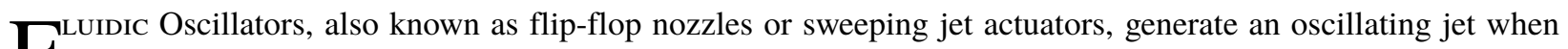
1 continuously supplied with a pressurized fluid. The internal flow is bistable and oscillates between two states. Figure 1(left) conceptually illustrates the internal dynamics of an oscillator which incorporates two feedback channels. This oscillator design is similar to the design investigated in the current study. Other types of oscillators exist (e.g., with only one feedback channel or without feedback channels) which involve different oscillation mechanisms. A more detailed review was presented by Gregory et al[1]. For the oscillator shown in figure 1, the jet enters the mixing chamber and attaches to either side. Part of the main jet is deviated into the feedback channel and guided back to the chamber inlet. Here, the flow interacts with the main stream and causes it to separate from the wall and to flip over to the other side of the mixing chamber where the process is re-initiated. This results in the oscillation of the emitted jet in a sweeping pattern. The photograph on the right side of figure 1 depicts the oscillating jet with water as a working fluid. Fluidic oscillators do not contain any moving parts. Therefore, they are attractive devices in numerous applications (i.e., windshield spray nozzles or shower heads)[2]. In recent years, fluidic oscillators have become of particular interest as flow control actuators. They have a proven high efficacy in a wide field of applications including combustion[3], noise[4] and separation control[5-7]. Despite the advantages and proven performance, the literature offers only limited information on the underlying mechanisms. Additional fluidic oscillator investigations are necessary to improve the understanding of flow phenomena inside and outside the oscillator and to validate numerical calculations enabling the optimization of these devices. For high-frequency naturally oscillating flow fields such as those emitted by fluidic oscillators, it is challenging to acquire time-resolved data, because the oscillation frequency exceeds the sampling rate of most available optical measurement systems such as particle image velocimetry (PIV). Therefore, phase-averaging of the recorded data is necessary. By using phase-averaging methods, all snapshots or signals within a certain interval are averaged. This yields a phase-averaged oscillation period and minimizes stochastic noise. Phase-averaging of a naturally oscillating flow field is not trivial due to the naturally fluctuating period lengths and the absence of external triggers. Various approaches to obtain phase-averaged data from an oscillating flow field have been pursued. One possibility is to force the oscillation with an external trigger[8]. However, this eliminates the self-sustaining natural oscillations and may affect natural flow phenomena. Other approaches attempt to lower the oscillation frequency by changing the working fluid or enlarging the oscillator[9, 10]. In these studies, signal conditioning[10] and mathematical methods[9] were used to phase-average the flow field.

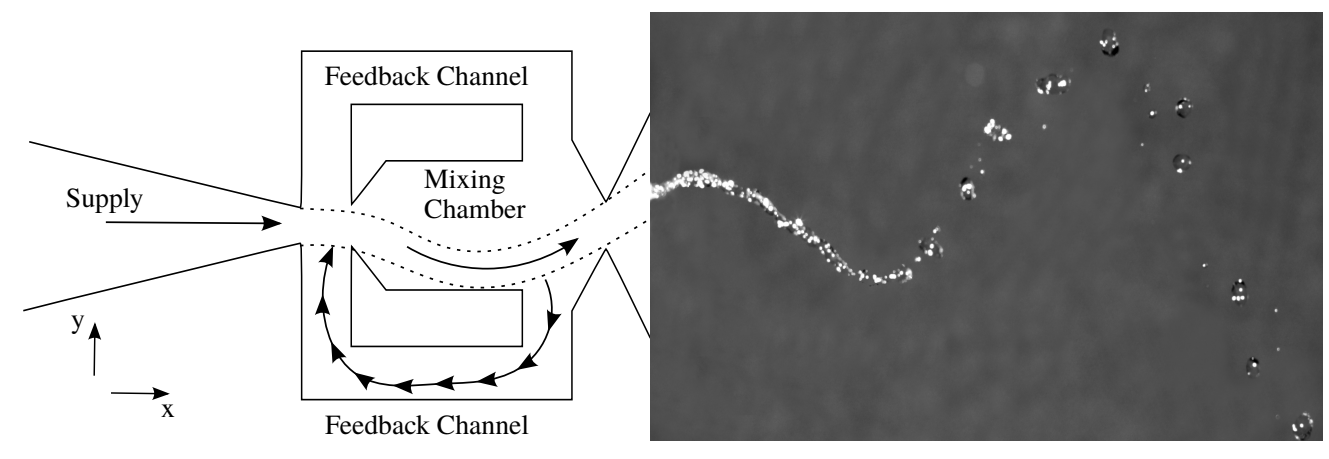

Fig. 1 Working principle of a fluidic oscillator.

The current study is aimed at examining various methods to obtain the time-resolved features of the internal and external flow field of a fluidic oscillator with a high quantitative accuracy. Experiments are conducted on a scaled-up fluidic oscillator similar to the design shown in figure 1 with the goal to establish a method applicable to higher jet velocities and oscillation frequencies. The advantages and disadvantages of these methods are evaluated and their 
accuracy is judged based on several criteria. The most suitable method is described in detail and applied to yield the time-resolved internal and external flow field. The results of the examined flow field are discussed in the preliminary work by Gärtlein et al.[11].

\section{Setup and Instrumentation}

The time-resolved study of fluidic oscillators is usually prevented by high oscillation frequencies and maximum sampling rates of the measurement equipment. In order to lower the oscillation frequency, previous experiments chose water as the working fluid [9]. In the present study, the oscillator is scaled-up and pressurized air is used as the supply fluid. The increased size lowers the frequency enabling time-resolved measurements of the internal and external flow field with a high-speed PIV system (figure 2). The scaled-up oscillator with an outlet area of $25 \mathrm{~mm} \times 25 \mathrm{~mm}$ is CNC machined from a solid piece of acrylic glass (PMMA). It is covered by a plate, which is verified to provide an airtight seal. The oscillator is mounted on a metal stand emitting its jet into an unobstructed, quiescent environment. The fluidic oscillator is supplied by a constant mass flow maintained by a digital controller by Bronkhorst ${ }^{\circledR}$ Mättig with a range of 0 to $100 \mathrm{~kg} / \mathrm{h}$ (accuracy: $\pm 0.6 \mathrm{~kg} / \mathrm{h}$ full scale). The area ratio from the plenum to the inlet of the oscillator is approximately 10:1. A honeycomb installed upstream of the inlet provides homogenous inflow conditions. The origin of the coordinate system is located at the center of the outlet nozzle.

Inside the oscillator, 55 pressure taps are symmetrically installed at selected positions based on the study by Bobusch et al.[9] Unused taps are sealed to prevent additional entrainment or leakage. The diameter and length of the pressure taps are optimized by using the calculation for the dynamic response of tubes[12]. This ensures time-resolved pressure measurements with insignificant phase delay, conservation of amplitude, and reduction of possible resonance effects. The diameter of the pressure taps is set to $0.8 \mathrm{~mm}$. Differential pressure sensors (HDO Series by Sensortechnics) with a range of 0 to 50 mbar (accuracy: $\pm 0.1 \mathrm{mbar}$ full scale) and a response rate of $10 \mathrm{kHz}$ are connected to the internal taps. A microphone and a hotwire probe may be positioned at the outlet of the oscillator. The PIV system includes one high-speed camera (Photron Fastcam SA1), one laser with an optical arm (Quantronix Darwin Duo 100 /Nd:YLF), a synchronizer (ILA Synchronizer), and an aerosol generator for oil droplets with an average diameter of $0.5 \mu \mathrm{m}$ (Palas AGF 10.0). This system is capable of recording up to 10,920 double frames with a maximum resolution of one mega pixel at a maximum sampling rate of $5 \mathrm{kHz}$. In this study a sampling rate of $1.5 \mathrm{kHz}$ is chosen, which is sufficient to acquire time-resolved flow field data. When supplied

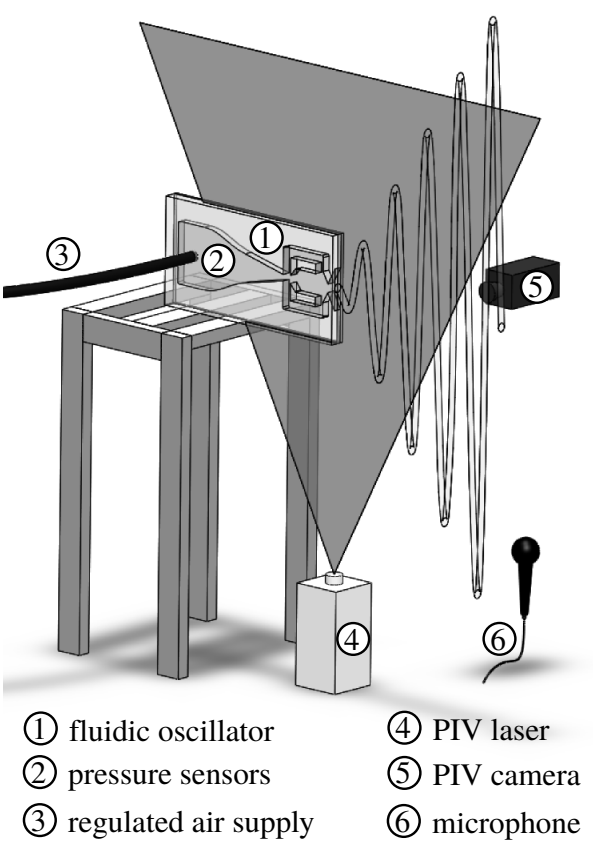

Fig. 2 Sketch of the experimental setup. with the highest available mass flow rate (i.e., $100 \mathrm{~kg} / \mathrm{h}$ ), the emitted jet oscillates at a maximum frequency of $23 \mathrm{~Hz}$ with an exit velocity of $U_{\text {outlet }}=37 \mathrm{~m} / \mathrm{s}$ (assuming incompressibility and ambient air properties). The pulse distance is adjusted according to the maximum velocity. The spatial resolution is approximately $2 \mathrm{~mm}$ with a maximum interrogation window size of $16 \times 16$ pixels and an overlap of $50 \%$. Measurements of pressure and PIV are executed simultaneously by two independent systems. If not noted otherwise, all investigated phase-averaging methods are applied to the same data set, which is obtained at an outlet velocity of $U_{\text {outlet }}=19 \mathrm{~m} / \mathrm{s}$. At this supply rate, the average oscillation frequency is $12.5 \mathrm{~Hz}$. In general, the trends are independent of the supply rate, if not a describing statement or figure is added. The investigated field of view is symmetric and covers the complete oscillation pattern. 


\section{Phase-Averaging Methods}

Various methods may be applied to phase-average a naturally oscillating flow field. These methods can be grouped into signal conditioning and mathematical methods. The signal conditioning methods require a time-resolved measurement signal which clearly identifies the oscillation periods. In contrast, mathematical methods correlate the phase between randomly acquired PIV snapshots. The general steps of both approaches are outlined in figure 3 . In subsequent sections, selected methods of both groups are described in detail. Additionally, the effect of the phase-averaging window size is discussed.

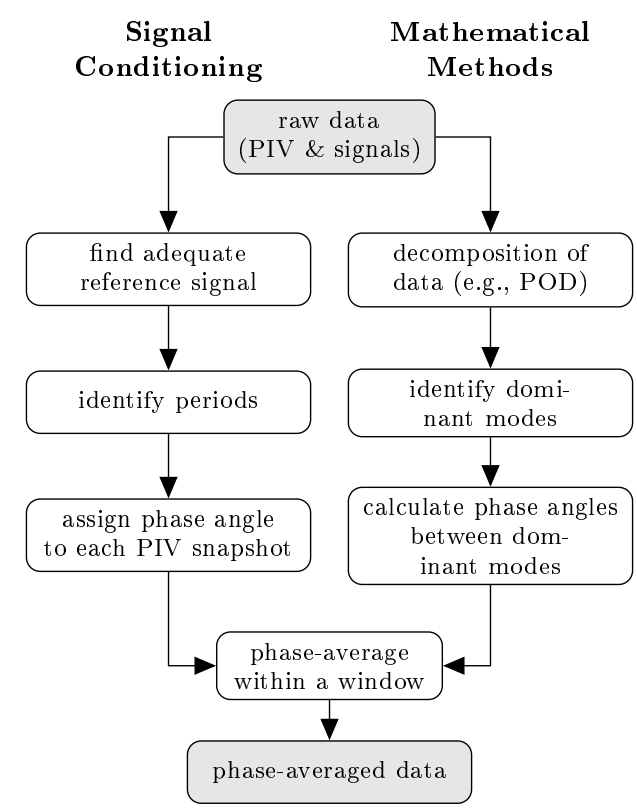

Fig. 3 General phase-averaging steps.

\section{A. Signal Conditioning Approaches}

It is possible to determine a phase angle for every time step by applying signal conditioning methods on a time-resolved reference signal which clearly reveals the individual oscillation periods.

\section{Possible Sources for a Reference Signal}

Various sources for a reference signal were investigated during this study. Hotwire anemometry, microphones, and pressure transducers have high possible sampling rates. However, the hotwire probe and microphone may present a disturbance to the outer flow field due to the required proximity to the outlet. Furthermore, external probes would be disadvantageous for experiments involving a free-stream. Optical measurement techniques (e.g., PIV) may be used as a potential reference signal because they do not disturb the flow field. However, they are often limited by low sampling rates making them insufficient for high-frequency oscillating flow fields. One exception is offered by Laser-Doppler-Velocimetry (LDV), which is capable of acquiring a reference signal at high sampling rates. However, simultaneous PIV measurements are conducted in this study yielding an undesirable complexity of the setup to avoid any potential interference between the PIV and LDV laser systems. As a solution, data from pressure taps inside the oscillator are chosen as reference signals. Resonance effects and phase delay in the pressure taps and ducts have to be considered for the design of the pressure tap geometry. Additionally, the source of the reference signal needs to be close to the flow field of interest to avoid phase-lags larger than one oscillation period. Otherwise, this may cause errors due to fluctuating period lengths. 


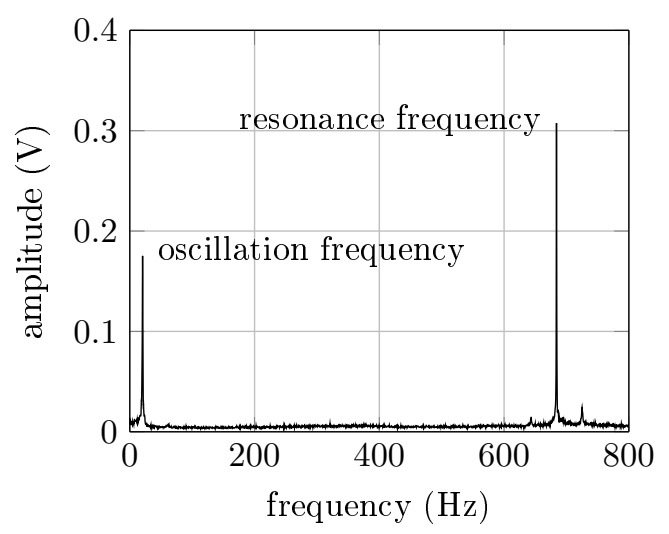

Fig. 4 Spectrum of the reference signal $\left(U_{\text {outlet }}=35 \mathrm{~m} / \mathrm{s}\right)$.

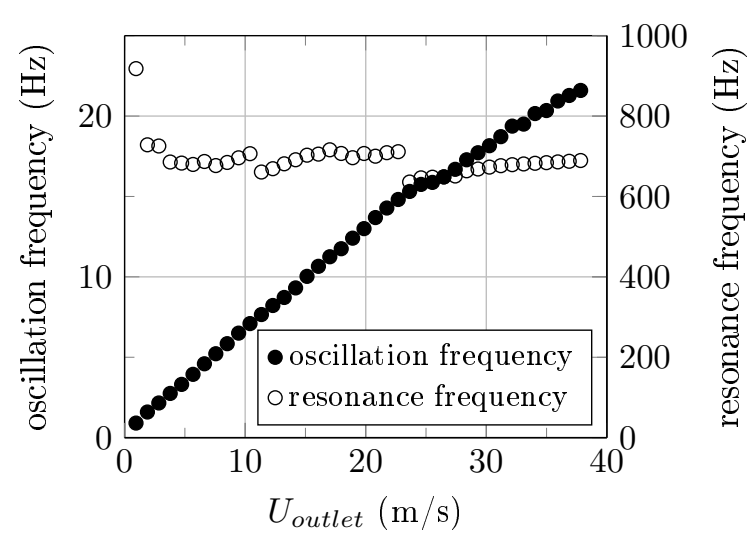

Fig. 5 Oscillation and resonance frequency as a function of the supply rate.

\section{Improvement of Signal Quality}

The quality of the reference signal is crucial for the correct identification of the oscillation periods. In this study, the reference signals from the pressure taps inside the feedback channels are dominated by a resonance phenomenon. The spectrum for a selected supply rate reveals two distinct peaks (figure 4). The first peak indicates the oscillation frequency whereas the second peak marks the resonance frequency. Figure 5 shows the resonance and oscillation frequency as a function of supply rate. It is evident that the oscillation frequency increases linearly with supply rate. In contrast, the resonance frequency remains approximately constant. This implies that the resonance frequency is only affected by the geometry. It is noteworthy that both the resonance frequency and oscillation frequency fluctuate around a mean value. The pressure measurements exhibit the mean resonance frequency at about $700 \mathrm{~Hz}$. If the feedback channel is acoustically modeled as a straight duct with open ends (duct length equals the length from feedback channel inlet to outlet), a standing wave is generated with a wavelength that is twice the duct length. The resulting frequency corresponds well to the observed resonance frequency. A cross-correlation between the pressure signals of both feedback channels reveals a phase relation between the resonance phenomena of both channels. The resonance can be either in phase or $180^{\circ}$ out of phase depending on the supply rate. The reasons for this dependence are not yet understood. A similar phenomenon was observed by Koso et al.[13]. The phase-averaged results do not extract the resonance effects because the oscillation frequency is one order of magnitude lower than the resonance frequency. Nevertheless, it is suspected that the resonance effects may increase the turbulence level inside the jet.

The quality of the reference signal is improved by subtracting the signals from two symmetrically positioned pressure taps (figure 6). This increases the amplitude of the oscillation because of the $180^{\circ}$ phase-lag between the feedback channels. A fifth order low-pass digital Butterworth filter is applied to the reference signal. The cutoff frequency is chosen in consideration of the oscillation frequency. The amplitude of the oscillation is preserved and a phase-lag is avoided by applying the filter forwards and backwards. It is noted that a constant phase-lag would not necessarily disturb the phase-averaging process because the beginning of the physical cycle is based on other criteria.

\section{Oscillation Period Identification}

After a proper reference signal is determined, the individual oscillation periods have to be identified. Although the pressure signal is chosen as a reference signal, the described period identification methods are applicable to all sources of a reference signal. The simplest period identification method requires subtracting the mean value from the reference signal and defining one period at every other change of sign (figure 7, top). However, this method (referred to 
Signal 1

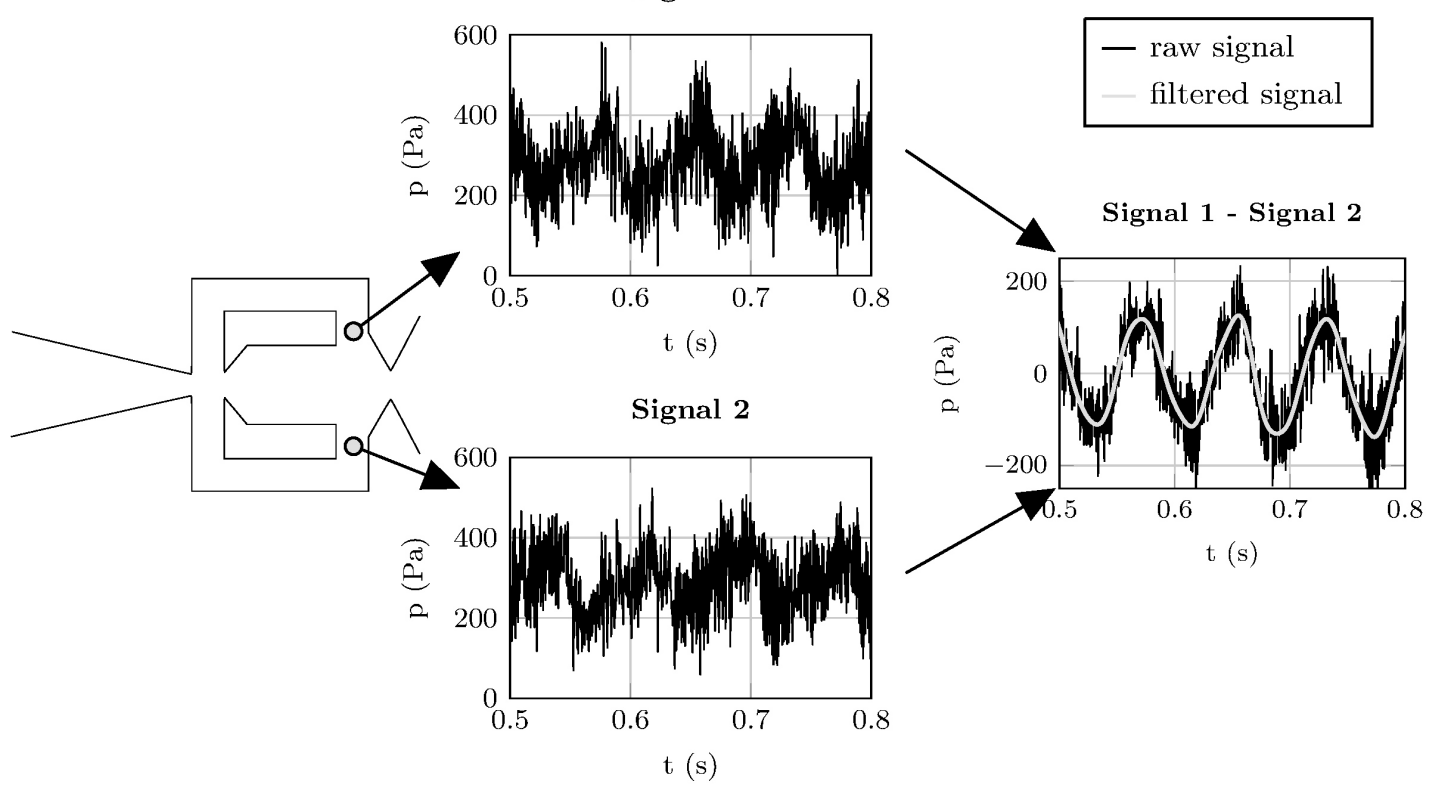

Fig. 6 Signal improvement by subtracting signals from two symmetrically aligned pressure taps.

as 'zero-crossing' method) is very susceptible to noise and amplitude fluctuations, which causes varying local mean values. For comparison with other phase-averaging methods, the 'zero-crossing' method is applied to a reference signal extracted from high-speed PIV data. Since this approach neither includes any mathematical methods modifying the data nor introduces any phase-lags due to the reference signal source, it yields a reliable result. The results are referred to as the 'benchmark method' in the following.
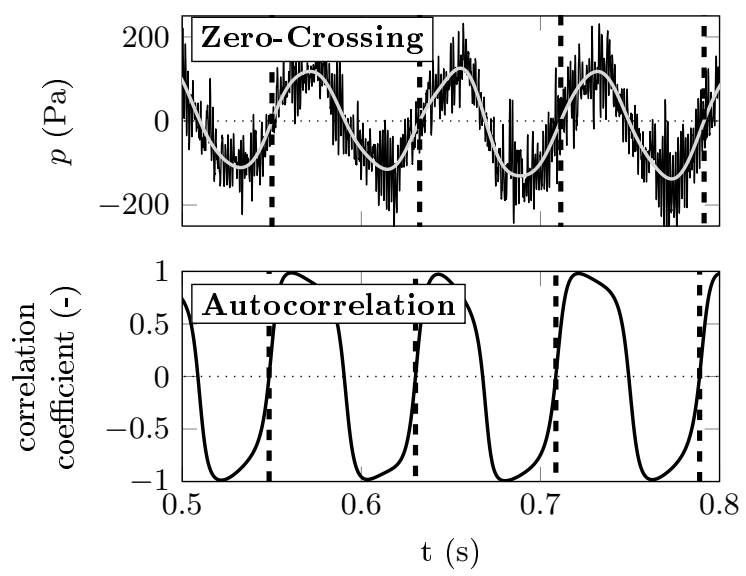

Fig. 7 Period identification in a reference signal (dashed: oscillation period starting points, dark: raw data (top) or correlation coefficient (bottom), light: filtered signal).

The drawback of the previously described 'zero-crossing' method is alleviated by applying an autocorrelation to the reference signal. A fragment of the reference signal is correlated with a shifting fragment of the same signal. This yields an oscillating correlation coefficient where every other change of sign marks a full period (figure 7, bottom). The autocorrelation method is less susceptible to local fluctuations, because the calculation of the correlation coefficient 
takes the local mean and root mean square (RMS) value into account. When applying the autocorrelation, two additional parameters become important and need to be examined: the size and the position of the fragment to be correlated. The position of the fragment is found to have no significant influence on the quality of the results. The size of the fragment defines the frequency of interest, but may also impose a dominant frequency upon the reference signal. Although the naturally oscillating flow field has a dominant frequency, deviations of the frequency are expected and have to be properly extracted by the phase-averaging algorithm. Figure 8 depicts the mean value and standard deviation of the individual oscillation periods with respect to the autocorrelated fragment size. Although the average period length is constant for a wide range of fraction lengths, the standard deviation changes. For small fragment lengths the correlation coefficient does not reveal the expected frequency, which is also evident in the plot of the average period length (figure 8). For large sample lengths, a decrease in the standard deviation is noticeable. This indicates an imposition of the dominant frequency and thus eliminates the natural fluctuations in the instantaneous period lengths. For a fragment length between 0.5 and 1.0 of the mean period length, a constant standard deviation is observed, which indicates unaffected periods while successfully isolating the frequency of interest. Although the standard deviation increases with the supply rate due to flow effects, this range of fragment lengths is found appropriate for all supply rates. It may change for other studies though and should always be investigated. In the following sections, a fragment length of one half average oscillation period is chosen. Alternatively, an iterative method using fragments of the phase-averaged reference signal for the autocorrelation of the next iterative step was investigated, but did not yield any improved quality. Another approach correlating a generic fragment with the reference signal also did not yield improved results.

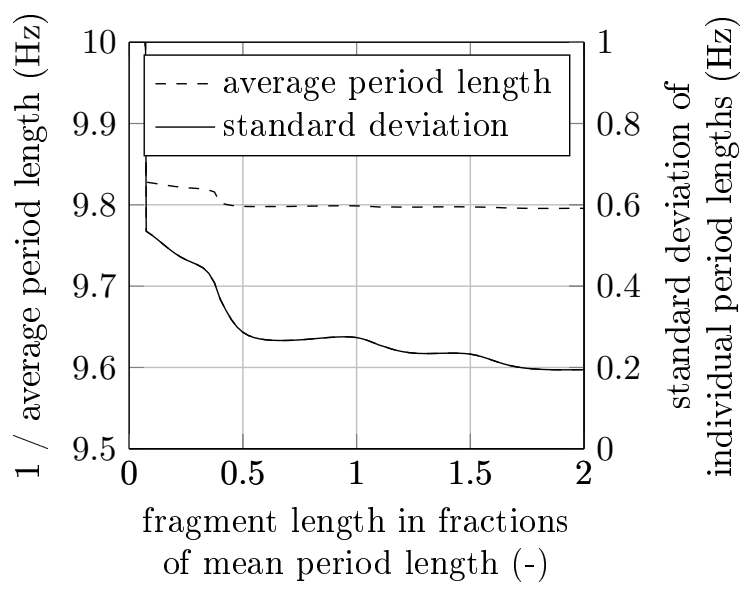

Fig. 8 Average individual period length and its standard deviation $\left(U_{\text {outlet }}=15 \mathrm{~m} / \mathrm{s}\right)$.

A challenge of using the autocorrelation method arises when comparing phase-averaged results. Due to applying the autocorrelation, the start of one oscillation period is not based on a physical criterion but rather on the choice of the signal fragment. In order to compare results, phase-aligning is necessary. This may be accomplished, for example, by comparing the phase-averaged reference signal. A phase-lag is determined by cross-correlating or finding the least difference between both phase-averaged signals. All phase-averaged pressure data and flow field results are then shifted to a common starting point.

\section{Determining the Instantaneous Phase Angles}

Both the 'zero-crossing' and the 'autocorrelation' method define the oscillation periods in the reference signal by identifying period starting points. The oscillation periods are then evenly divided into a number of phase angle windows where the number of windows depends on the window size. The influence of this parameter is discussed in 
section III-C. Snapshots are assigned to the appropriate phase angle window based on their time stamp (Eq. 1). All snapshots within one phase angle window are averaged yielding a phase-averaged oscillation period. The accuracy of the described methods is increased by using the half-period starting points because this yields a finer resolution of the natural fluctuations.

$$
\phi=180^{\circ} \cdot \frac{\text { time stamp - appropriate half-period starting time }}{\text { instantaneous half-period duration }}+\left\{\begin{array}{cl}
0^{\circ}, & \text { if first half-period } \\
180^{\circ}, & \text { if second half-period }
\end{array}\right.
$$

\section{B. Mathematical Methods}

Two suitable mathematical methods for phase reconstruction of a naturally oscillating flow are selected and described in this section. The first method applies the Hilbert transformation[14, 15] to a reference signal and the second method employs proper orthogonal decomposition[16, 17] (POD) directly to the PIV snapshots.

\section{Hilbert Transformation}

The Hilbert transform is used to calculate the instantaneous phase angle and frequency. It is defined over an integral using the Cauchy principal value and can be calculated using a fast Fourier transform (FFT) and an inverse Fourier transform (Eq. 2).

$$
F(H(g))(\omega)=F(g)(\omega)(-i \cdot \operatorname{sgn}(\omega))
$$

$H(g)$ is the Hilbert transform of $g, F(g)$ the Fourier transform of $g$, and $\operatorname{sgn}(\omega)$ is the signum function. The Hilbert transform can be interpreted as a phase shifter, which shifts a sinusoidal function with a positive frequency by $-90^{\circ}$. By applying the Hilbert transform to a time-resolved measurement signal $g(t)$, the analytical signal $g_{a}$ can be written as Eq. 3.

$$
g_{a}=g+i \cdot H(g)
$$

With this analytical signal the phase can be reconstructed by calculating the angle between the real and imaginary part (Eq. 4).

$$
\phi=\arctan (H(g) / g)
$$

Hence, the instantaneous phase angle $\phi$ and frequency can be calculated with the Hilbert transformation for an arbitrary time signal $g$. This phase angle is assigned to the simultaneously acquired PIV snapshots which are then phase-averaged accordingly. The quality of the results is improved by applying a fifth order digital Butterworth low-pass filter (cutoff frequency $=2 \cdot$ oscillation frequency) to the reference signal, as the Hilbert transformation is very sensitive to noise. In this study, this phase-averaging method is called 'Full Hilbert'. The Hilbert transformation may be extended by an empirical mode decomposition which decomposes the reference signal into intrinsic mode functions prior to applying the regular Hilbert transform. This method is called the Hilbert-Huang transformation[18], but is not investigated in this study. 


\section{Proper Orthogonal Decomposition}

The second mathematical approach employs the method of proper orthogonal decomposition (POD), which is mainly used to detect coherent structures. POD decomposes a field into its unique modes sorted by their energy contents. The velocity $u(x, t)$ can be decomposed into the mean velocity $u_{0}$ and the sum of the products of the modes $\Phi_{i}(x)$ with their time dependent modal coefficients $a_{i}(t)$ for every instance in time (Eq. 5).

$$
u(x, t)=u_{0}+\sum_{i=1}^{N} a_{i}(t) \Phi_{i}(x)
$$

The residual of this approximation is minimized if the sum of the square deviation is minimized. This corresponds to minimizing the residual kinetic energy and leads to an eigenvalue problem (Eq. 6).

$$
\mathrm{R} \Phi_{i}=\lambda_{i} \Phi_{i}
$$

$\mathrm{R}$ is the correlation matrix of the velocity fluctuations and the eigenvalues $\lambda_{i}$ are twice the kinetic energy $u^{2}$ captured by each mode. In practice, the modes and the coefficients can be calculated with a singular value decomposition. The modes are sorted by descending content of energy. Bobusch et al.[9] suggested that the flow field of the fluidic oscillator oscillates harmonically between the first two modes (i.e., modes with the most energy content) with a phase shift of approximately $90^{\circ}$. Accordingly, a phase angle for each PIV snapshot can be calculated with the corresponding time dependent modal coefficients $a_{1}(t)$ and $a_{2}(t)$ (Eq. 7), which are normalized by their respective eigenvalues.

$$
\phi=\arctan \left(\frac{a_{2} \sqrt{2 \lambda_{1}}}{a_{1} \sqrt{2 \lambda_{2}}}\right)
$$

Thus, the phase angle is calculated by employing the POD directly to the flow field without the need for a reference signal. In fact, the PIV snapshots are not required to have equally spaced timestamps. Additional details on the mathematical background of this method are described by Berkooz et al.[16] and Legrand et al.[17, 19]

\section{Possible Improvements}

Both the Hilbert transformation and the POD may be used to determine the phase angle for each PIV snapshot. In section IV-A it is shown that these mathematical methods have drawbacks in correctly reconstructing the time information within a period. Improvements may be achieved by using only the period starting point information and evenly distributing the phase angles within one period similar to the approach described in section III-A. However, time-resolved data is required for this approach. The Hilbert transformation is applied to a time-resolved reference signal. Thus, it may be used to identify the period starting points. This approach is referred to as 'Partial Hilbert' in the following sections. The POD is not capable of identifying period starting points because it is generally applied to data with insufficient sampling rates. In section IV-B additional means of improving the accuracy of the POD method without the need for time-resolved data are explored.

\section{Influence of the Averaging Window Size}

In order to obtain phase-averaged results, a phase angle range must be defined wherein all apposite snapshots are averaged. The angle range is referred to as the averaging window size. The window size is defined by its total angle size and the according result is assigned to the median phase angle (e.g., a total window size of $10^{\circ}$ for a phase angle of $0^{\circ}$ refers to $\phi=0^{\circ} \pm 5^{\circ}$ ). The choice of window size must balance the reduction of signal noise with the preservation of inherent flow characteristics. In order to determine the optimum window size, an RMS value is considered. Here, the 
phase-averaged velocity field obtained for a particular window size is subtracted from the phase-averaged result of the next larger window size. For each phase angle, the standard deviation of the entire flow field difference is calculated. Spatially and temporally averaging the standard deviation yields a value which is not representative of the flow field, but it is used for judging the quality of the results. The increment in window size is constant for this analysis. The respective RMS value is expected to obtain its minimum if the most flow effects are retained with minimal noise. According to this approach, the optimum total window size for $U_{\text {outlet }}=19 \mathrm{~m} / \mathrm{s}$ is approximately $3^{\circ}$, yielding an average of 91 snapshots per phase-averaging window (figure 9). It was found that the relative approximate error is less than $3 \%$ for more than 50 snapshots per window, which confirms the convergence of the averaging process. For the sake of simplification, this window size is chosen for all supply rates. The optimum window size does vary with changing supply rates within a small range, which is most likely caused by differing signal quality. Any additional increase in the temporal resolution of the phase-averaged data is limited by the data quality, because the flow features which are to be resolved eventually obtain the same order of magnitude as the noise.

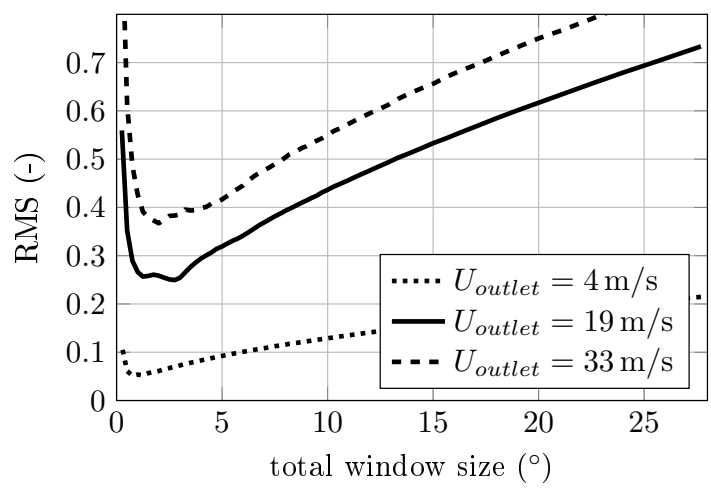

Fig. 9 Optimizing the averaging window size (autocorrelation method of differential pressure signal).

The averaging of all snapshots collected in one window may yield reduced amplitudes due to potentially substantial changes within the respective time frame. In order to increase the retained velocity amplitude, Zilberman et al.[20] applied an iterative approach. The snapshots within a window are cross-correlated with the respective phase-averaged result. The snapshot data is then shifted accordingly and phase-averaged again until the changes in the results reach a convergence criterion. This approach was also pursued in the current study. However, due to already small window sizes, the signal noise is of the same order as any possible shift. Therefore, this iterative method did not yield any improved results.

\section{Results}

In this section, the previously described phase-averaging methods are evaluated for their accuracy in revealing the natural flow field. The comparison is based on several criteria (i.e., velocity field data, unsteady oscillation frequency, and snapshot distribution). Significant discrepancies are observed with POD, thus this method is examined separately and modified to yield improved results. The advantages and disadvantages of all methods are summarized and one selected procedure is employed to accurately reveal the phase-averaged internal and external flow field of a fluidic oscillator.

\section{A. Comparison between Methods}

A comparison between the described phase-averaging methods is challenging because no exact results of the naturally oscillating flow field are available for proper validation. A visual examination of the velocity distribution may be the first step. In figure 10 the phase-averaged results as produced by the discussed methods are shown. The 
velocity plot is extracted from one point on the center line of the flow field where, due to symmetry, the fluctuation frequency is doubled. Consequently, the gradients are higher and discrepancies between the individual results are more pronounced. The same results are found at other positions as well. All results are phase-aligned by using the $v$-component of the phase-averaged velocity obtained using the benchmark method (i.e., zero-crossing method with a PIV signal as a reference signal). Although the qualitative behavior of the oscillation is properly covered by all methods, substantial quantitative differences are evident. The amplitude of the velocity is a dominant indicator for the accuracy of a phase-averaging method because averaging the wrong snapshots causes a significant loss in amplitude. The small amplitudes obtained by the benchmark method indicate the poor quality of the phase-averaging algorithm. In contrast, the amplitudes of the velocity magnitude for the other methods are equal and at the same phase angle. Especially the autocorrelation and both Hilbert methods show a remarkable agreement. The results of the POD differ from the others regarding the temporal duration of the velocity peaks. The reasons for these quantitative deviations are discussed separately in the following section because POD is frequently applied in other research to unsteady flow fields. Another possibility is the investigation of the RMS value of all snapshots averaged within a phase window. Higher RMS values indicate a larger deviation from the mean value and are indicators for incorrect phase angle allocation. However, some methods such as POD minimize the RMS value within a window by maximizing the variance between individual modes. Evidently, this does not necessarily yield the 'true' solution.

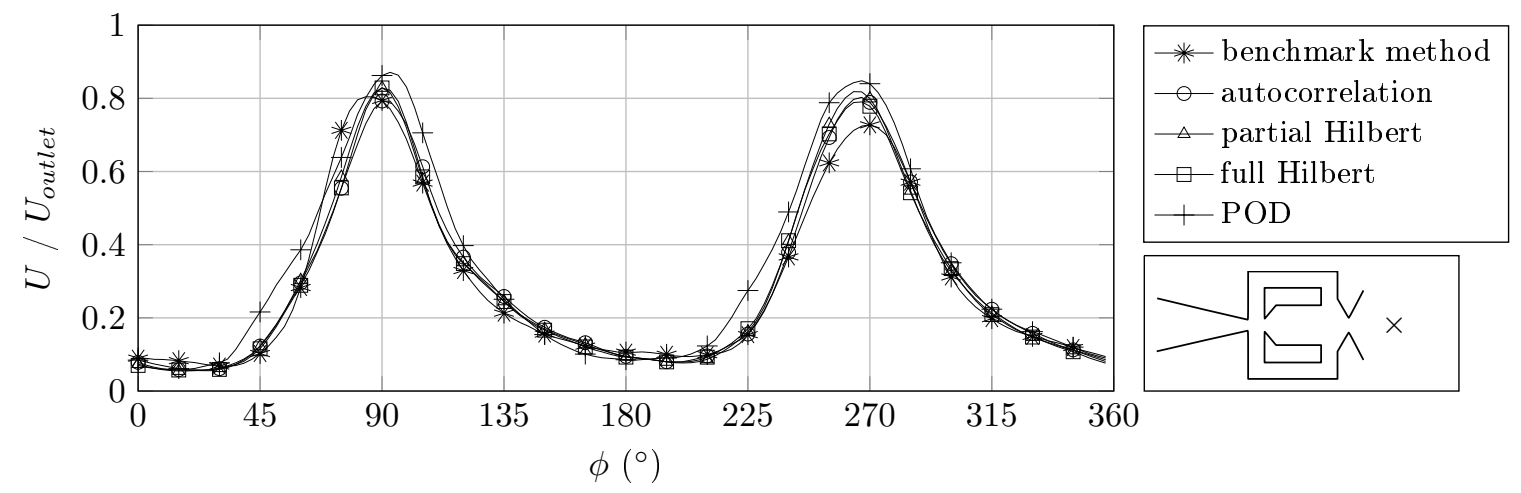

Fig. 10 Phase-averaged velocity profiles at $\left(x / d_{h}=4, y / d_{h}=0\right)$. Every fifth data point marked.

In addition to the visual examination, two additional approaches to compare methods are pursued in this study. The first approach compares the duration of the individual oscillation periods. The individual periods are identified based on zero-crossing from $359^{\circ}$ to $0^{\circ}$ for all methods except for the full Hilbert method. The individual periods are used to evaluate a resulting period length. For the full Hilbert method, the instantaneous frequency determined by the Hilbert transformation is used. In a naturally oscillating flow field, the period length is expected to fluctuate. The method yielding the least period length fluctuations may be considered most accurate. The probability density of the local period lengths for each method is presented in figure 11. The average period length is approximately equal for all methods which supports their general applicability. However, the full Hilbert method generates broad fluctuations in the instantaneous period length. This is likely caused by a non-sinusoidal reference signal which results in fast changing instantaneous frequencies. The least variation in period length is evident for the autocorrelation, benchmark, and POD method. 


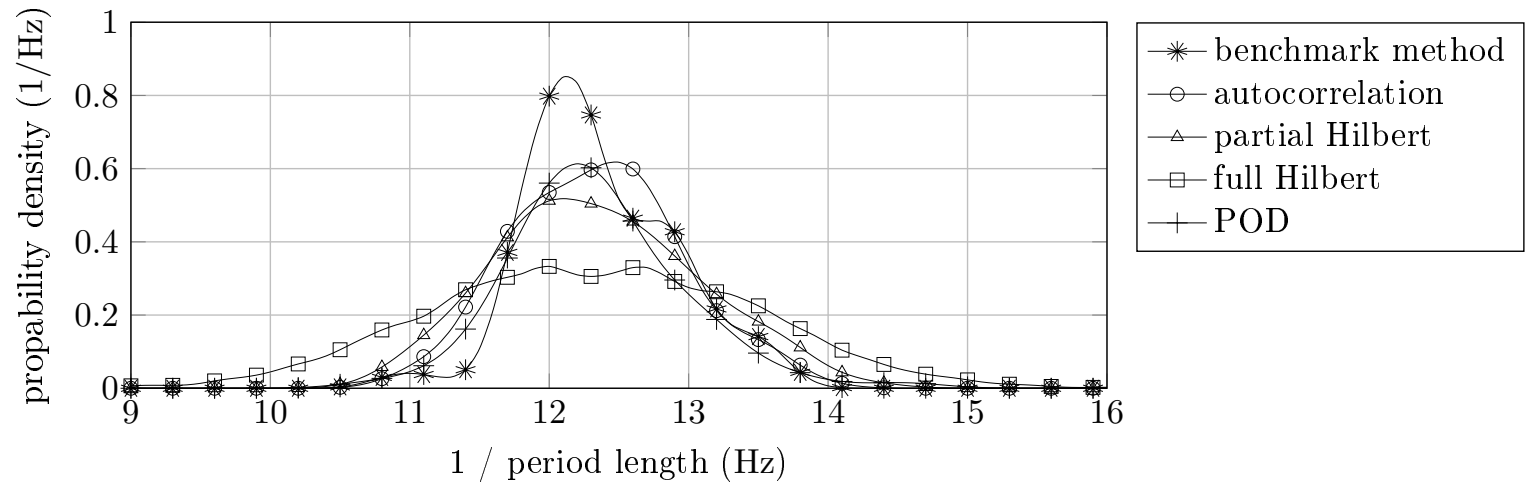

Fig. 11 Probability density of individual period length for various methods.

The comparison discussed in figure 11 addresses the correct identification of the oscillation periods. Furthermore, in a naturally oscillating flow field the snapshots should be evenly distributed over all phase angle windows of one period. Recall that 10,920 snapshots are recorded at a constant sampling rate of $1.5 \mathrm{kHz}$. This is another possibility to ensure the accuracy of the phase-averaging methods. In figure 12 it is evident that the full Hilbert method and especially the POD have some drawbacks in the accurate allocation of phase angles. This observation is consistent for all supply rates except for the full Hilbert method. The phase angle allocation for this method is improved for higher supply rates because more oscillation periods are covered within the reference signal. It is noteworthy that the range of phase angles with high velocity magnitude (figure 10) corresponds to the phase angle windows with the least number of snapshots for the POD method. Consequently, the high velocities are stretched over a wider range of phase angles. At other locations within the flow field, the properties are stretched/compressed accordingly. Possible sources of this problem for the POD are investigated in section IV-B. Figure 12 also reveals that the snapshots per phase angle are evenly distributed for all methods which identify a period oscillation start. This is expected due to the definition of the phase angle assignment.

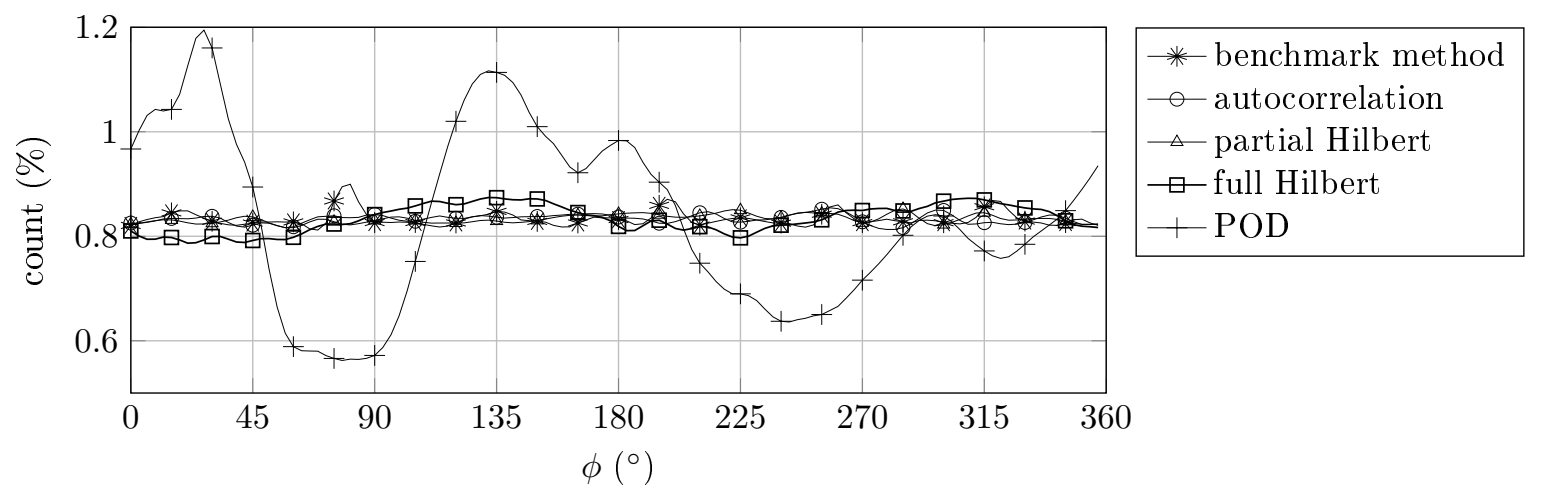

Fig. 12 Distribution of snapshots per phase angle window. Every fifth data point marked.

It can be concluded that according to figure 10, the benchmark method does not sufficiently phase-average the data because it decreases the velocity amplitudes. In fact, the period identification method 'zero-crossing' is highly sensitive to noise, making it a poor choice for most applications. Furthermore, the reference signal extracted from PIV is not suitable for high oscillation frequencies due to the usually low sampling rates of optical measurement methods. POD and full Hilbert have some drawbacks in allocating the correct phase angle, which reduces the accuracy of the phase-averaged results (figures 11 and 12). The phase-averaging, based on conditioning a reference signal extracted from pressure signals, is found to yield reliable results in this study. Nevertheless, due to the susceptibility to signal noise, applying an autocorrelation is required. This must be handled carefully in order to avoid the imposition of a 


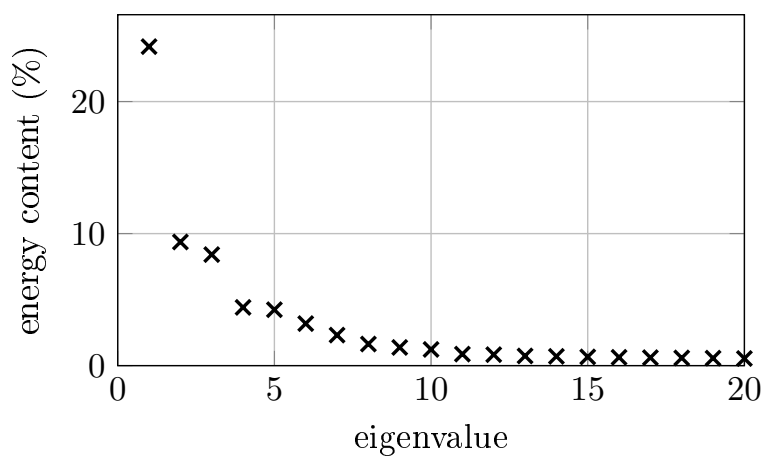

Fig. 13 Energy content of the eigenvalues.

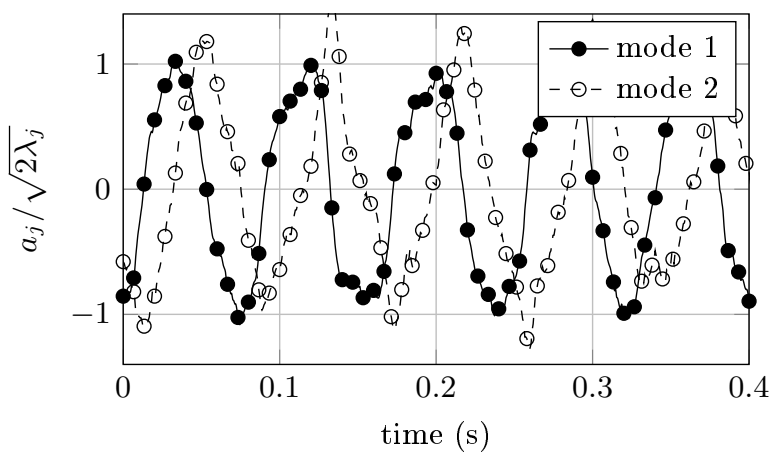

Fig. 14 Time-dependent mode coefficients. Every tenth data point marked.

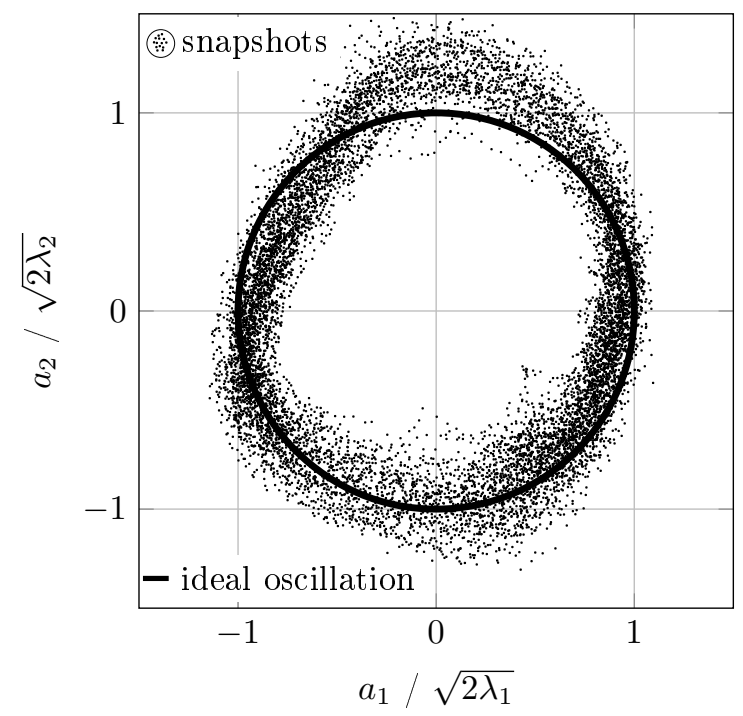

Fig. 15 Phase portrait of modal coefficients $a_{1}(t)$ and $a_{2}(t)$ of the external flow field.

dominant frequency. The partial Hilbert method may also be a good alternative. This method defines a period starting point at the extreme values of a period where low gradients cause the method to be susceptible to noise. The POD is of particular interest for studies where no reference signal is available. This motivates the discussion of potential improvements in the subsequent section.

\section{B. Challenges with POD and According Modifications}

The POD method is frequently used for examining and phase-averaging unsteady flow fields. [9, 21-23] It is a very attractive tool for phase-averaging flow fields because it requires neither a reference signal nor time-resolved snapshots of the flow field. Despite these advantages, POD has limitations in reconstructing the correct oscillation period of the investigated flow field causing quantitative deficiencies. Due to the general interest in POD, the shortcomings of this method are investigated in more detail. A modification is also suggested which significantly improves the accuracy of the POD approach.

Phase-averaging using POD requires the calculation of phase angles between dominant modes, which are normalized by their respective eigenvalues (Eq. 7). For an ideal harmonic oscillation between two modes, the modes exhibit approximately equal energy content, the time-resolved normalized mode coefficients are sinusoidal, and they have a $90^{\circ}$ 
phase shift. In the case of a fluidic oscillator, mode one has more than twice the energy of mode two (figure 13). The time-resolved normalized mode coefficients are shown in figure 14 . Although a $90^{\circ}$ phase-shift is evident, the mode coefficients show discrepancies. The amplitude of the normalized mode 2 coefficient is higher than the amplitude of the mode 1 coefficient. Additionally, the shape of the mode coefficients differs. It is noteworthy that the differences between the individual oscillation periods are higher for mode two than for mode one. These observations lead to a distorted phase portrait (figure 15). Consequently, the phase angle calculation (Eq. 7) leads to a periodic distortion of the flow field properties, thereby reducing the accuracy of the POD method. The described observations for the POD modes are likely caused by oscillating velocity amplitudes at the exit and decreasing velocity magnitude downstream of the exit due to entrainment. Moreover, information may be lost because the higher modes are neglected for the phase angle allocation. In order to improve the results, a modification is suggested by imposing a constant distribution of snapshots per phase angle window. This assumption was previously justified based on a constant sampling rate in conjunction with a naturally fluctuating flow field. It may be assumed that although the allocated phase angle value is erroneous, the correct phase angle sequence is revealed by the POD. Instead of phase-averaging the snapshots according to their determined phase angle, the snapshots are sorted sequentially by these phase angles and evenly allocated to a predetermined number of phase angle windows. In this study all 10,920 snapshots are sorted in ascending order based on their assigned phase angle. A window size of $3^{\circ}$ yields 120 phase angle windows within one oscillation period. Therefore, 91 snapshots per phase angle window are desired to obtain an equal distribution of snapshots per phase angle window. Based on this, the first 91 snapshots are assigned to the first phase angle window, the next 91 snapshots to the second phase angle window, and so forth until all phase angle windows have the same amount of snapshots available for phase-averaging. In order to incorporate all available snapshots and to avoid a minor phase delay, the number of snapshots should be a multiple of the number of phase angle windows. The modified approach imposes an even distribution of snapshots while maintaining the POD determined temporal sequence of the snapshots. Note that this modified POD is only applicable for snapshots which are randomly distributed throughout the oscillation periods.

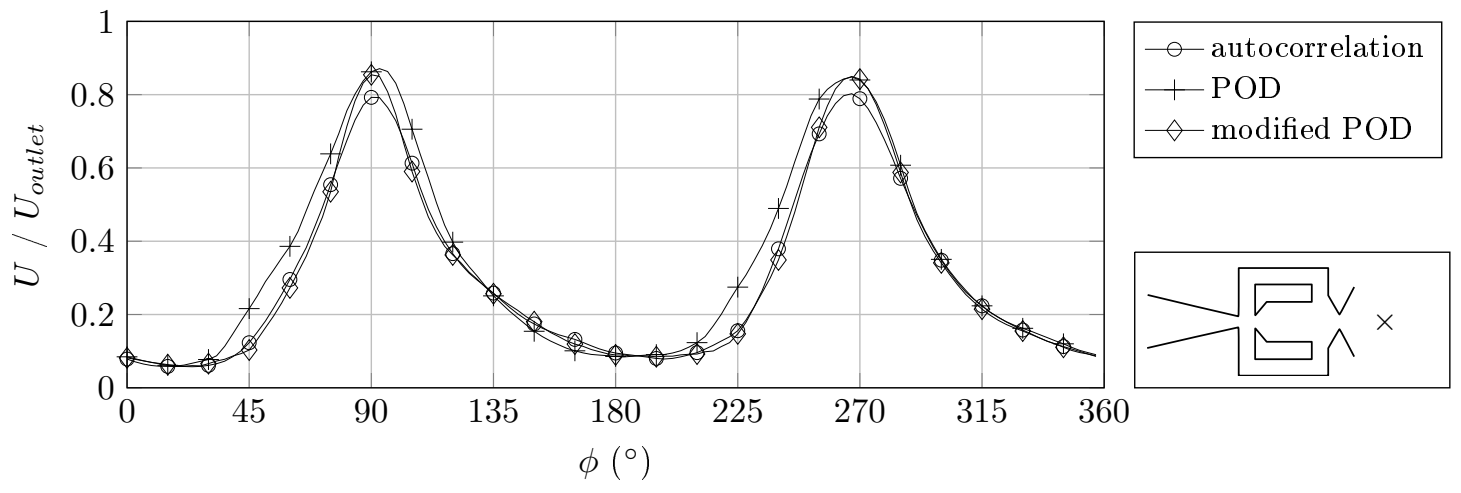

Fig. 16 Phase-averaged velocity profiles at $\left(x / d_{h}=4, y / d_{h}=0\right)$. Every fifth data point marked.

In figure 16 the phase-averaged results of the conventional and modified POD method are compared to the autocorrelation method at the same position as in figure 10. As expected, the conventional POD approach yields deviations. In contrast, the modified POD shows good agreement with the autocorrelation method. This is noteworthy because the two methods are based on completely different approaches (i.e., mathematical vs. signal conditioning method). This result assures confidence that both methods are accurate in revealing the naturally oscillating flow field. In fact, the modified POD yields even higher amplitudes of the phase-averaged velocity, indicating a better phase-averaged result. Nevertheless, some problems with the modified POD method are identified. Various regions of the flow field may be recorded separately in order to improve the spatial resolution. The POD methods encounter problems especially in flow regions where the oscillation is not fully captured. It is suspected that the flow field patterns 
caused by the oscillation of the jet are either incomplete or not dominant enough for proper identification by the POD methods. Consequently, the POD methods are only applicable if the complete oscillation cycle is covered within one acquired field of view. If this condition is met, the modified POD method provides a simple and accurate tool in future studies and to other researchers.

\section{Summarized Comparison and Applied Method}

The previous sections describe and compare various phase-averaging methods. Each method is accompanied by certain advantages and disadvantages which are summarized in table 1. Based on various criteria, the methods which yield the most accurate results in this study are identified.

Table 1 Summary of the investigated phase-averaging methods.

\begin{tabular}{|c|c|c|c|}
\hline Method & Description & Advantages & Disadvantages \\
\hline Benchmark method & $\begin{array}{l}\text { - applied to time-resolved PIV } \\
\text { signal } \\
\text { - period starting points identi- } \\
\text { fied by zero-crossing of veloc- } \\
\text { ity } v \text {-component }\end{array}$ & $\begin{array}{l}\text { - reliable results due to sim- } \\
\text { plicity }\end{array}$ & $\begin{array}{l}\text { - } \text { susceptible to variations in lo- } \\
\text { cal mean values } \\
\text { - high sampling rate of PIV mea- } \\
\text { surements required }\end{array}$ \\
\hline Autocorrelation & $\begin{array}{l}\text { - signal fragment correlated with } \\
\text { time-resolved reference signal } \\
\text { - period starting point identified } \\
\text { by zero-crossing of correlation } \\
\text { coefficient }\end{array}$ & $\begin{array}{l}\text { - insensitive to noise } \\
\text { - yields most promising results } \\
\text { in this study }\end{array}$ & $\begin{array}{l}\text { - may impose a dominant oscil- } \\
\text { lation frequency depending on } \\
\text { fragment length }\end{array}$ \\
\hline Partial Hilbert & $\begin{array}{l}\text { - applied to filtered time- } \\
\text { resolved reference signal } \\
\text { - period starting points identi- } \\
\text { fied by zero-crossing of instan- } \\
\text { taneous phase angle }\end{array}$ & $\begin{array}{l}\text { no imposing of a dominant } \\
\text { oscillation frequency }\end{array}$ & $\begin{array}{l}\text { - sensitive to noise } \\
\text { - based on locating extreme val- } \\
\text { ues within the signal }\end{array}$ \\
\hline Full Hilbert & $\begin{array}{l}\text { - applied to filtered time- } \\
\text { resolved reference signal } \\
\text { - yields instantaneous phase an- } \\
\text { gle for every data point }\end{array}$ & $\begin{array}{l}\text { - assignment of instantaneous } \\
\text { phase angle to every snap- } \\
\text { shot without additional pro- } \\
\text { cessing }\end{array}$ & $\begin{array}{l}\text { - deficits in reconstructing the } \\
\text { correct time information } \\
\text { - sensitive to noise }\end{array}$ \\
\hline POD & $\begin{array}{l}\text { - mode decomposition of every } \\
\text { PIV snapshot } \\
\text { - phase angles obtained directly } \\
\text { from modal coefficients }\end{array}$ & $\begin{array}{l}\text { - no time-resolved data neces- } \\
\text { sary } \\
\text { - only PIV snapshots required }\end{array}$ & $\begin{array}{l}\text { - incorrect phase angle alloca- } \\
\text { tion due to uneven energy con- } \\
\text { tents between modes one and } \\
\text { two }\end{array}$ \\
\hline Modified POD & $\begin{array}{l}\text { - mode decomposition of every } \\
\text { PIV snapshot } \\
\text { - based on phase angle se- } \\
\text { quence, snapshots are evenly } \\
\text { distributed over desired num- } \\
\text { ber of windows }\end{array}$ & $\begin{array}{l}\text { - no time-resolved data neces- } \\
\text { sary, only PIV snapshots re- } \\
\text { quired } \\
\text { - phase-averaging directly ap- } \\
\text { plied to local data field }\end{array}$ & $\begin{array}{l}\text { - only applicable when oscil- } \\
\text { latory behavior is dominant } \\
\text { within data field } \\
\text { - if more PIV regions are ac- } \\
\text { quired, phase-alignment and } \\
\text { transition between the regions } \\
\text { may be challenging }\end{array}$ \\
\hline
\end{tabular}

It is found that all approaches are suitable for phase-averaging the flow field of the fluidic oscillator. However, some methods have drawbacks in quantitative accuracy (e.g., the full Hilbert and conventional POD method). The autocorrelation and modified POD method yield the most accurate results in this study. Although the modified POD approach has the great advantage of not requiring time-resolved data, it may have problems with flow fields not covering the entire oscillation and sufficiently capturing the dominant structures. Furthermore, phase-aligning and combination 
of various flow fields without an overlap may be challenging. Therefore, a reference signal in combination with the autocorrelation approach is chosen for phase-averaging the flow field data of the fluidic oscillator in this study. The applied processing steps are summarized in the following overview:

1) At the beginning of the phase-averaging process, a proper reference signal must be found. In this study, pressure signals from the feedback channel, the outlet, and the outer region of the fluidic oscillator are suitable to meet the requirements. Further improvements in signal quality are achieved by subtracting two signals from symmetrically positioned pressure taps and applying a digital low-pass filter.

2) The reference signal is autocorrelated with an extracted sample size from one half to one full expected period length. The sign change of the correlation coefficient defines the start of each half oscillation period. Afterward, the half oscillation cycle is divided into equally sized phase angle windows. The simultaneously acquired PIV snapshots are assigned to the appropriate window by evaluating their time stamps.

3) All phase angles within a $3^{\circ}$ range are averaged.

This procedure is successfully employed in order to phase-average the internal and external flow field of a fluidic oscillator as illustrated in figure 17. The time-resolved flow field allows a detailed investigation of various instantaneous flow field properties (e.g., jet velocity, jet width, deflection angle, and entrainment). The preliminary results of this investigation are presented by Gärtlein et al.[11].
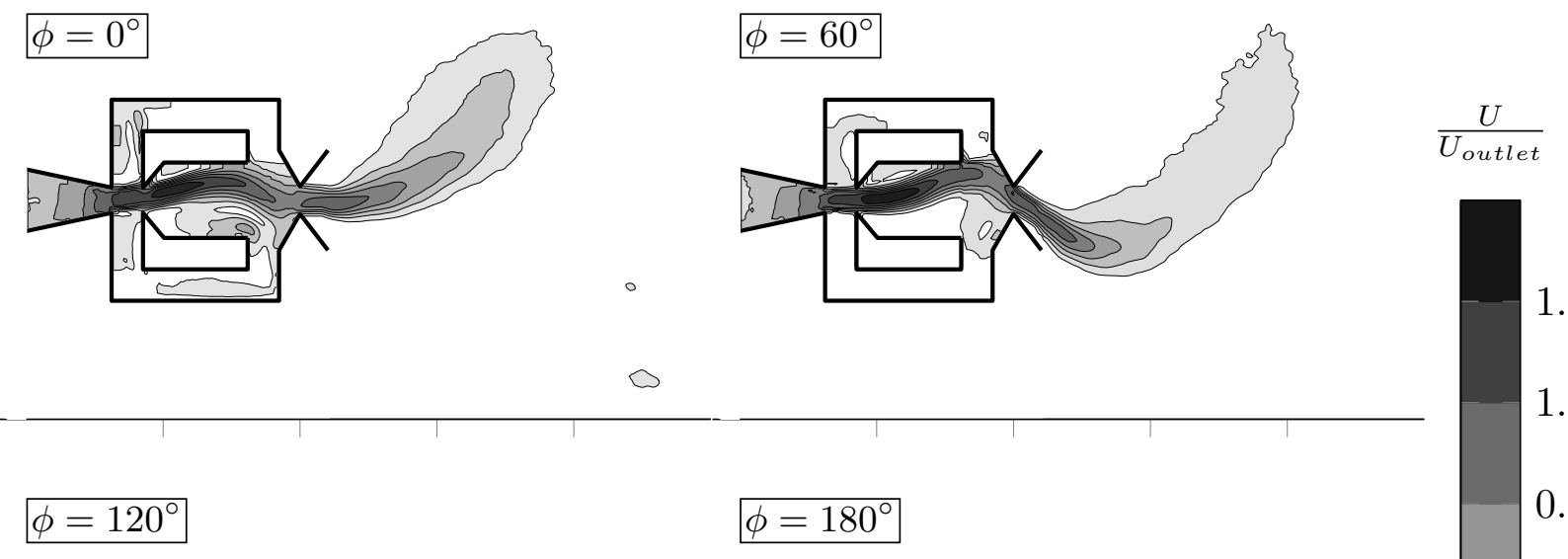

$\phi=120^{\circ}$

$$
\phi=180^{\circ}
$$
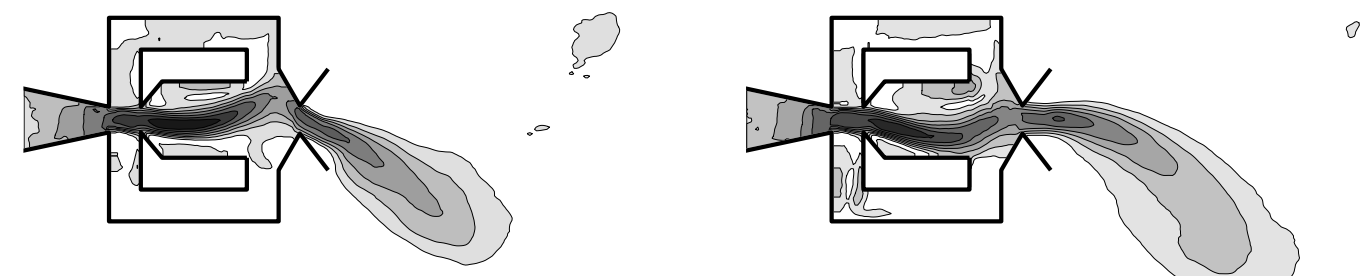

$$
\begin{array}{llll}
-5 & 0 & 5 & 10 \\
& x / d_{h} &
\end{array}
$$

$$
\begin{array}{llll}
-5 & 0 & 5 & 10 \\
& x / d_{h} &
\end{array}
$$

Fig. 17 Phase-averaged flow field. 


\section{Conclusion}

Various methods for phase-averaging the natural flow field from a fluidic oscillator are analyzed. They are grouped into mathematical and signal conditioning methods. Selected methods are discussed in detail and evaluated for their accuracy based on several criteria. These criteria include the minimum fluctuation in instantaneous period length, the conservation of velocity amplitudes, and the number of snapshots per phase-averaging window. All methods are found to be suitable for phase-averaging the data, however with differing accuracy. The POD shows drawbacks in the correct temporal reconstruction of the external flow field of the oscillator. It is found that the POD artificially alters the time information by stretching and shrinking phases of the oscillation. A modified POD approach is introduced which imposes an even distribution of snapshots per phase angle window by using the phase angle sequence determined by the conventional POD. Although based on completely different approaches, the modified POD method yields excellent agreement with other methods involving a time-resolved reference signal. This agreement provides additional confidence that the modified POD is capable of revealing the naturally oscillating flow field of the fluidic oscillator. This modified POD method is a simple and accurate phase-averaging tool without requiring time-resolved data and may also be a suitable tool for other studies investigating oscillating flow fields. However, the POD method does have limitations in identifying the dominant modes if the oscillatory pattern is not fully covered within the field of view. In this study, a method also suitable for phase-averaging a flow field comprised from various PIV sections is desired. Additionally, phase-aligning is challenging because of the absence of a common signal if no overlap between the sections is present which is the case for the acquisition of three-dimensional flow fields. These reasons led to the pursuit of a phase-averaging method based on conditioning a time-resolved reference signal. After evaluating the advantages and disadvantages of the investigated approaches, the autocorrelation method is chosen. The subtracted signal between two symmetrically aligned pressure taps inside the feedback channels is used as the reference signal. This signal is digitally filtered and an autocorrelation is employed for the accurate identification of the oscillation periods. The PIV snapshots are averaged within a prescribed window size which is optimized with regards to minimizing noise while maintaining flow features. This procedure is successfully applied to yield the time-resolved internal and external flow field of a fluidic oscillator. It is also suitable for oscillating flow fields with higher velocities and oscillation frequencies. Furthermore, a conventional PIV system with lower sampling rates may be employed as long as a simultaneously recorded reference signal is obtainable and resolved in time. By acquiring the pressure signal inside the oscillator, other experiments such as the interaction of the jet with an external stream may be analyzed with this method as well.

\section{Acknowledgments}

The authors thankfully acknowledge the helpful discussions and assistance of the team at the Hermann-FöttingerInstitut. Especially the input of Moritz Sieber was highly appreciated. The authors also thank Dr. Jesse Little and Joshua Gray for the editorial review of the paper.

\section{References}

[1] Gregory, J., and Tomac, M. N., "A Review of Fluidic Oscillator Development," AIAA 43rd Fluid Dynamics Conference, 2013. doi:10.2514/6.2013-2474.

[2] Stouffer, R., "Oscillating spray device," Patent US 4151955, 1979. URL http://www.patentlens.net/patentlens/ patent/US_4151955/en/.

[3] Guyot, D., Paschereit, C. O., and Raghu, S., "Active Combustion Control Using a Fluidic Oscillator for Asymmetric Fuel Flow Modulation,” International Journal of Flow Control, Vol. 1, No. 2, 2009, pp. 155-166. doi:10.1260/175682509788913335.

[4] Raman, G., and Raghu, S., "Cavity Resonance Suppression Using Miniature Fluidic Oscillators," AIAA Journal, Vol. 42, No. 12, 2004, pp. 2608-2612. doi:10.2514/1.521. 
[5] Seele, R., Tewes, P., Woszidlo, R., McVeigh, M. A., Lucas, N. J., and Wygnanski, I. J., "Discrete Sweeping Jets as Tools for Improving the Performance of the V-22," AIAA Journal of Aircraft, Vol. 46, No. 6, 2009, pp. 2098-2106. doi:10.2514/1.43663.

[6] Woszidlo, R., and Wygnanski, I. J., "Parameters Governing Separation Control with Sweeping Jet Actuators," AIAA 29th Applied Aerodynamics Conference, 2011. doi:10.2514/6.2011-3172.

[7] Woszidlo, R., Stumper, T., Nayeri, C., and Paschereit, C. O., "Experimental Study on Bluff Body Drag Reduction with Fluidic Oscillators," 52nd Aerospace Sciences Meeting, 2014. doi:10.2514/6.2014-0403.

[8] Gregory, J. W., Gnanamanickam, E. P., Sullivan, J. P., and Raghu, S., "Variable-Frequency Fluidic Oscillator Driven by a Piezoelectric Bender," AIAA Journal, Vol. 47, No. 11, 2009, pp. 2717-2725. doi:10.2514/1.44078.

[9] Bobusch, B. C., Woszidlo, R., Bergada, J. M., Nayeri, C. N. N., and Paschereit, C. O., "Experimental study of the internal flow structures inside a fluidic oscillator," Experiments in Fluids, Vol. 54, No. 6, 2013. doi:10.1007/s00348-013-1559-6.

[10] Wassermann, F., Hecker, D., Jung, B., Markl, M., Seifert, A., and Grundmann, S., "Phase-locked 3D3C-MRV measurements in a bi-stable fluidic oscillator," Experiments in Fluids, Vol. 54, No. 3, 2013. doi:10.1007/s00348-013-1487-5.

[11] Gaertlein, S., Woszidlo, R., Ostermann, F., Nayeri, C., and Paschereit, C. O., "The Time-Resolved Internal and External Flow Field Properties of a Fluidic Oscillator,” 52nd Aerospace Sciences Meeting, 2014. doi:10.2514/6.2014-1143.

[12] Bergh, H., and Tijdeman, H., Theoretical and experimental results for the dynamic response of pressure measuring systems, Nationaal lucht-en ruimtevaartlaboratorium, 1965.

[13] Koso, T., Kawaguchi, S., Hojo, M., and Hayami, H., "Flow Mechanism of a Self-Induced Oscillating Jet Issued from a Flip-Flop Jet Nozzle," JSME-KSME Fluids Engineering Conference, Vol. 5, 2002.

[14] Wlezien, R. W., and Way, J. L., "Techniques for the Experimental Investigation of the Near Wake of a Circular Cylinder," AIAA Journal, Vol. 17, No. 6, 1979, pp. 563-570. doi:10.2514/3.61178.

[15] Luo, H., Fang, X., and Ertas, B., "Hilbert Transform and Its Engineering Applications," AIAA Journal, Vol. 47, No. 4, 2009 , pp. 923-932. doi:10.2514/1.37649.

[16] Berkooz, G., Holmes, P., and Lumley, J. L., "The Proper Orthogonal Decomposition in the Analysis of Turbulent Flows," Annual Review of Fluid Mechanics, Vol. 25, No. 1, 1993, pp. 539-575. doi:10.1146/annurev.fl.25.010193.002543.

[17] Legrand, M., Nogueira, J., and Lecuona, A., "Flow temporal reconstruction from non-time-resolved data part I: mathematic fundamentals," Experiments in Fluids, Vol. 51, No. 4, 2011, pp. 1047-1055. doi:10.1007/s00348-011-1111-5.

[18] Huang, N. E., and Wu, Z., "A review on Hilbert-Huang transform: Method and its applications to geophysical studies," Reviews of Geophysics, Vol. 46, No. 2, 2008. doi:10.1029/2007rg000228.

[19] Legrand, M., Nogueira, J., Tachibana, S., Lecuona, A., and Nauri, S., "Flow temporal reconstruction from non time-resolved data part II: practical implementation, methodology validation, and applications," Experiments in Fluids, Vol. 51, No. 4, 2011, pp. 861-870. doi:10.1007/s00348-011-1113-3.

[20] Zilberman, M., Wygnanski, I., and Kaplan, R. E., “Transitional boundary layer spot in a fully turbulent environment,” Physics of Fluids, Vol. 20, No. 10, 1977, p. S258. doi:10.1063/1.861739.

[21] Oudheusden, B. W. v., Scarano, F., Hinsberg, N. P. v., and Watt, D. W., "Phase-resolved characterization of vortex shedding in the near wake of a square-section cylinder at incidence," Experiments in Fluids, Vol. 39, No. 1, 2005, pp. 86-98. doi:10.1007/s00348-005-0985-5.

[22] Perrin, R., Braza, M., Cid, E., Cazin, S., Barthet, A., Sevrain, A., Mockett, C., and Thiele, F., "Obtaining phase averaged turbulence properties in the near wake of a circular cylinder at high Reynolds number using POD," Experiments in Fluids, Vol. 43, No. 2-3, 2007, pp. 341-355. doi:10.1007/s00348-007-0347-6.

[23] Oberleithner, K., Sieber, M., Nayeri, C. N., O.Paschereit, C., Petz, C., Hege, H.-C., Noack, B. R., and Wygnanski, I. J., "Three-dimensional coherent structures in a swirling jet undergoing vortex breakdown: stability analysis and empirical mode construction," Journal of Fluid Mechanics, Vol. 679, 2011, pp. 383-414. doi:10.1017/jfm.2011.141. 